\title{
THE NATURE AND IMPLICATIONS OF THE RESWITCHING OF TECHNIQUES *
}

\author{
Michael Bruno, Edwin Burmeister, and Eytan Sheshinski
}

I. Introduction, 526. - II. Alternative discrete capital models, 528. III. Reswitching in two-good technologies, 531.- IV. Reswitching in a general capital model, 538. - V. Some additional implications for economic theory, 546 .

\section{INTRODUCTION}

By reswitching of techniques we mean the recurrence at different rates of interest of a whole matrix of activities or a "technique of production." The "Ruth Cohen Curiosum" may be considered a special case where only a single activity recurs. ${ }^{1} \mathrm{We}$ have analyzed the conditions under which reswitching can occur, which are perfectly general, the conditions under which it cannot occur, which are quite restrictive, and some of the important implications for capital theory.

In a paper read to the Econometric Society meeting in Rome in September 1965, Luigi Pasinetti ${ }^{2}$ was the first to question seriously the validity of Levhari's nonswitching theorem. ${ }^{3}$ It is this challenge that gave us the immediate inspiration for our own work. However, Pasinetti's earlier Rome discussion seemed incomplete to us because there was no clear indication whether or not Levhari's

* We are greatly indebted to Paul A. Samuelson and Robert M. Solow for their helpful leads, insights, and numerous discussions. Frank M. Fisher and Robert M. Solow have also given us very helpful comments on an earlier draft.

Likewise we are indebted to the Center for International Affairs, Harvard University, for partial support of M. Bruno's research, to the National Science Foundation for partial support of E. Burmeister's research under Grant GS-571 and to the Irwin Foundation for a Fellowship supporting E. Sheshinski's Ph.D. thesis at Massachusetts Institute of Technology, of which his present contribution forms a part.

1. Joan Robinson, Accumulation of Capital (London: Macmillan, 1956); Piero Sraffa, Production of Commodities by Means of Commodities (Cambridge: Cambridge University Press, 1960); and M. McManus, "Process Switching in the Theory of Capital," Economica, XXX (May 1963). The reswitching phenomenon has also been discussed by Michio Morishima in "Mrs. Robinson's New Book and Professor Leontief's Dynamic System," mimeographed paper, Feb. 1958; "Refutation of the Nonswitching Theorem," this Journal, this issue; and Equilibrium, Stability, and Growth (Oxford: Clarendon Press, 1964), p. 126.

2. Luigi L. Pasinetti's present paper "Changes in the Rate of Profit and Switches of Techniques," this Journal, this issue, is a revised version of the paper he presented in Rome.

3. David Levhari, "A Nonsubstitution Theorem and Switching of Techniques," this Journal, LXXIX (Feb. 1965), 98-105. 
theorem was in fact wrong and therefore the primary issue was still unresolved. 4 In our subsequent work we have individually and jointly discovered in various ways that the theorem is indeed wrong; the credit, however, goes to Pasinetti's lead. Pasinetti's present paper is a considerable modification and revision of his earlier analysis and now touches on a number of aspects which we have ourselves analyzed in the meantime. We shall nonetheless risk minor repetition for the sake of clarification and proceed with our own discussion as originally envisaged.

Numerical examples and the realization that switching points are roots of $n$-th degree polynomials (and therefore numerous) have convinced us that reswitching may well occur in a general capital model. A seemingly small alteration in the fundamental lemma can be shown to make Levhari's theorem and its original proof formally correct, but unfortunately the class of cases for which it would remain valid is thereby restricted quite heavily. We have analyzed some alternative sufficiency conditions for nonswitching which are of interest in themselves, especially for the two-sector case, but such analyses only help to convince one of their highly restrictive nature.

Two points must be clarified at the outset: (1) There is no essential difference between the circulating-capital and fixed-capital models as far as the important capital-theoretic issues are concerned. One, in fact, includes the other as a special case. (2) Indecomposability of the technique matrix is essentially irrelevant for the reswitching discussion. A short digression on the various capital models and a clarification of their relationships follows in Section II of this paper.

In Section III we use Samuelson's two-sector canonical model of capital to show that there are simple classes of cases in which both reswitching and no reswitching can occur, and we give simple sufficiency conditions for either to take place. It is also shown that in any $n$-sector model having only one capital good, reswitching cannot occur; difficulties arise with two capital goods. There follows a simple two-sector numerical example to serve as a definite proof that reswitching can occur.

In Section IV we discuss the $n$-sector model, showing that in general there can be $n$ switching points between any two techniques. We then use Descartes' rule of signs to formulate and interpret a

4. E.g., the modified numerical counterexample produced by Pasinetti in his Rome paper did not satisfy the indecomposability on the whole technique matrix as required by the conditions of Levhari's theorem. 
sufficiency condition for nonswitching, which turns out to be extremely restrictive in nature.

Once the reswitching phenomenon is acknowledged, it is important to realize its implications for capital theory. As is often the case after the fact, one finds it hard to differentiate between reswitching and another well-known phenomenon, namely the existence of multiple rates of return to investment in present value calculations.

Perhaps the most interesting and most important finding of our analysis concerns the behavior of consumption and the rate of interest (profit) in steady states. The reswitching phenomenon implies the existence of "perverse behavior" where it is not true that steady-state consumption always rises as the rate of interest falls. ${ }^{5}$ Rather for certain ranges of the interest rate, steady-state consumption may rise when the rate of interest rises. Moreover, although the reswitching phenomenon alerted us to this possibility, we find that such "perverse behavior" can exist even when no reswitching occurs on the factor-price frontier. The latter and related issues form the subject of Section V.

\section{Alternative Discrete Capital Models}

Various discussions of the reswitching phenomenon employ a great variety of capital models differing with respect to such assumptions as depreciation, timing of wage payments, and the time structure of inputs in the production of capital goods. Pasinetti has illustrated his arguments with a Sraffa model in which capital goods essentially consist of "maturing" labor inputs at different time periods; Levhari considered another Sraffa model with one-period circulating capital. ${ }^{6}$ Naturally, one prefers to think in terms of more general fixed-capital models which include the former as special cases. Since all of these alternative models basically lead to the same theoretical conclusions, it seems useful to begin our discussion with a short classification. We try to minimize confusion by indicating how they all relate to each other and how the present issue can be discussed in terms of any of the models and therefore is best analyzed in greatest generality. An issue somewhat related to the same question is the irrelevance of decomposability or indecomposability of the technology matrix.

5. The possibility of such "perverse behavior" was pointed out without proof by Professor Morishima in Equilibrium, Stability, and Growth, op. cit., p. 126.

6. Pasinetti, op. cit., Levhari, op. cit., and Sraffa, op. cit. 
1. Consider an $n$-sector fixed-proportions technology $\left(a_{i j}\right)$ using and producing $n$ capital goods (prices $P_{j}$ ) and using one primary factor, labor $\left(a_{o j}\right)$ commanding a nominal wage $w$.

Denoting the depreciation rate for the $j$-th capital good by $\mu_{j}$ and the rate of interest by $r$, and supposing wages are paid during the production period, we have:

$$
P_{j}=w a_{o j}+\sum_{i=1}^{n}\left(\mu_{i}+r\right) P_{i} a_{i j} ; j=1, \ldots, n,
$$

or in vector notation

$$
p=a_{o}[\mathrm{I}-\rho a]^{-1}
$$

where $p=\left(\frac{P_{j}}{w}\right)$ and $\rho=[\mu+r]$, a diagonal matrix whose $i$-th diagonal entry is $\left(\mu_{i}+r\right)$. We must assume that the maximal interest rate, $r_{a}^{*}$, is positive and that $a_{o j} \geqslant 0$ to have $p_{j} \geqslant 0$.

There are some obvious special cases of this model which are used often and which we shall at times mention. All of these involve the assumption of a uniform depreciation rate:

$$
\mu_{i}=\mu \text { and } \rho=r+\mu, \text { a scalar. }
$$

The two extreme subcases here are:

$$
\mu=0, \quad \rho=r \text { (capital is infinitely durable), }
$$

and

$$
\mu=1, \quad \rho=r+1 \text { (there is only circulating capital). }
$$

2. Next suppose we slightly alter our above assumptions and have wages paid at the beginning of the production period. The only change required in equations (II.2) is that a factor $(1+r)$ now must multiply $a_{o}$, i.e., we have

$$
p=(1+r) a_{o}[I-\rho a]^{-1} .
$$

Now if we consider the special case $\mu=1(\rho=r+1)$, we get the Sraffa circulating-capital model used by Levhari, i.e.,

$$
p=a_{o}[\lambda I-a]^{-1} \text { where } \lambda \equiv \frac{1}{1+r} .
$$

Other than a factor $(1+r)$ due to a different assumption about wage payments, there is no real difference between the two models except that (II.1) is more general. Clearly the nonsubstitution theorem as well as the discussion of the switching problem could be conducted equally well in terms of the fixed-capital model.

3. Now suppose there are some goods that take more than one period to produce. One can either treat goods-in-process of different ages as different goods (with different activities) or else calculate directly the implied price relationships. For example, if a commodity available at present requires an input $a_{o j}$ of labor $t$ periods 
earlier, then that input's contribution to present cost must be $w a_{o j}(1+r)^{t}$. The Pasinetti-Sraffa numerical example uses precisely the latter type of capital model. Clearly a further generalization would have not only labor but also capital inputs required in earlier periods. All of these ideas could be incorporated without difficulty in a modified expression for (II.1) or (II.2). For simplicity we shall confine our discussion to the models that can be derived from (II.2) or (II.3).

There is one general common characteristic of all these models from which reswitching and other properties can be shown to follow. Under some quite unrestrictive assumptions, ${ }^{7}$ we can always expand the price vector (in terms of labor units) as a convergent power series in $r$, i.e.,

$$
p=g_{o}+g_{1} r+g_{2} r^{2}+\ldots+g_{n} r^{n}+\ldots
$$

where $g_{i}$ are nonnegative vectors whose elements eventually approach zero as $n$ increases. (II.5) is the equation of the factor-price frontier (FPF) in terms of $p$ instead of $1 / p$. In Section IV we shall have occasion to examine (II.5) for the general fixed-capital model (II.1) and to provide the economic interpretation of the $g_{i}$ 's. Let us only note here that these vectors can be interpreted in terms of the direct and indirect use of labor in production. The price of any product is thus positive if it uses directly or indirectly some of the primary factor labor. ${ }^{8}$ As long as one of these vectors (for $i \geqslant 1$ ) is not zero, we obtain a downward sloping factor-price frontier i.e., $\frac{d p}{d r}>0$, from (II.5). At the same time we cannot generally say whether the FPF when expressed as $1 / p(r)$ will be convex or concave unless we know something about the coefficients. ${ }^{9}$

Finally consider another generalization. Suppose that in addition to fixed-capital goods and consumption goods, we have Leontief-type intermediate goods in the system. We propose to show that the formal properties of the system (II.2) remain unchanged.

Suppose that in addition to a fixed-capital matrix $a$ there is an ordinary input-output matrix $\bar{a}$ (assume $a$ and $\bar{a}$ are defined so as

7. Some variant of the Hawkins-Simon conditions. matrix.

8. This, incidentally, does not require indecomposability of the technique

9. $\frac{d^{2}(1 / p)}{d r^{2}}=-p^{-2}\left(\frac{d^{2} p}{d r^{2}}\right)+2 p^{-3}\left(\frac{d p}{d r}\right)$. Even though $\frac{d^{2} p}{d r^{2}}>0$ so that $p(r)$ will always be convex to the origin, $\frac{d^{2}\left(\frac{1}{p}\right)}{d r^{2}}$ is a difference of two positive terms and may be either positive or negative. 
to be conformable)..$^{1}$ The price equations must now be modified as follows:

$$
\text { (II.2') } \quad p=a_{o}[I-\bar{a}-\rho a]^{-1} \text {. }
$$

But this can also be written in the form

Now define

$$
p=a_{o} S[I-\rho a S]^{-1} \quad \text { where } S=[I-\bar{a}]^{-1} .
$$

$$
\begin{array}{ll}
A_{o}=a_{o}[I-\bar{a}]^{-1} & \text { (= "total" labor input) } \\
A=a[I-\bar{a}]^{-1} & \text { (= "total"capital matrix) }
\end{array}
$$

and we have

$$
p=A_{o}\left[I-{ }_{\rho} A\right]^{-1}
$$

which is formally equivalent to (II.2).

This derivation has two corollaries. First, for purposes of exposition we can ignore the existence of intermediate inputs since they do not alter the formal structure of the capital model. The second, somewhat more interesting, is that there is a sense in which the distinction between decomposable and indecomposable models is artificial. For example, suppose that the "total" capital matrix $A$ is decomposable while the input-output matrix $\bar{a}$ is indecomposable; then it may be misleading to term the model either decomposable or indecomposable. This ambiguity should be an additional indication that decomposability is not an important property in the present context. ${ }^{2}$

\section{Reswitching in Two-Good Technologies}

Before returning to a more general discussion of $n$-sector models, it seems helpful to use a simpler two-sector technology to illustrate the switching problem.

Consider a technique $a$ producing two goods, a capital good (subscript 1) and a consumption good (subscript 2). The capital good is assumed to depreciate at a fixed rate $\mu_{a}$, and labor (subscript 0 ) is the only primary input. If we denote the fixed coefficients for this technique by $a_{i j}(i=0,1 ; j=1,2)$ and express the wage rate $w$ and price of capital good $P_{K}$ in terms of the consumption good as numéraire, we get the following price equations: ${ }^{3}$

1. See, e.g., Morishima, Equilibrium, Stability, and Growth, op. cit., Chaps. III and IV, and Jacob T. Schwartz, Lectures on the Mathematical Method in Analytical Economics (New York: Gordon and Breach, 1961), Chap. I.

2. Samuelson has suggested an alternative argument using the fact that any decomposable capital matrix can be turned into an indecomposable one by adding some arbitrarily small elements in the right places thereby causing only an infinitesimal change in the FPF.

3. This so-called canonical model was introduced by Samuelson, "Parable and Realism in Capital Theory: The Surrogate Production Function," Review of Economic Studies, XXIX (June 1962), 193-207. 
(III.1) $\quad P_{K}=a_{01} w+a_{11}\left(r+\mu_{a}\right) P_{K}$

(III.2) $\quad 1=a_{02} w+a_{12}\left(r+\mu_{a}\right) P_{K}$,

from which the following equation for the factor-price curve $F P C_{a}$ (Figure I) can be derived:

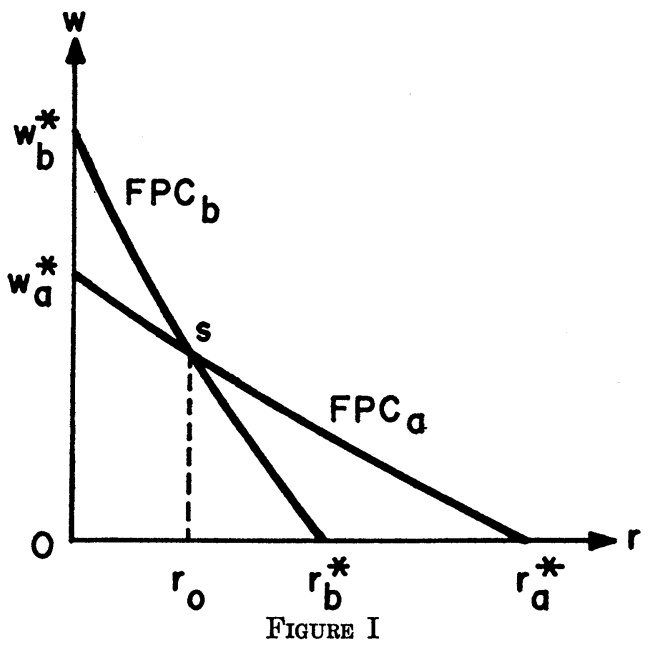

(III.3)

$$
w=\frac{1-\left(\mu_{a}+r\right) a_{11}}{a_{02}+\left(\mu_{a}+r\right) G_{a}}
$$

where

$$
G_{a}=\operatorname{det}\left[\begin{array}{ll}
a_{01} & a_{02} \\
& \\
a_{11} & a_{12}
\end{array}\right] .
$$

This downward sloping FPC will be convex or concave to the origin according to whether $G_{a}>0$ or $<0$, namely according to whether the consumption good industry is more capital-intensive or more labor-intensive than the capital good industry. For our purposes this distinction is unimportant, and the curve in Figure $I$ is drawn with the assumption $G_{a}>0$. Next we note that the FPC intersects both the $w$ and the $r$ axes at $w_{a}^{*}$ and $r_{a}^{*}$ respectively, where:

$$
w_{a}^{*}=\frac{1-\mu_{a} a_{11}}{a_{02}+\mu_{a} \mathrm{G}_{a}}=\begin{gathered}
\text { "net" } 4 \text { labor productivity in the con- } \\
\text { sumption good sector }
\end{gathered}
$$

4. "Net labor productivity" is the quantity of consumption goods produced per unit of labor after taking into account the indirect labor embodied in the current replacement cost of capital. Perhaps this interpretation can be seen better by rewriting this expression in the form:

$$
w_{a}^{*}=\frac{1}{a_{02}+\frac{a_{01} \mu_{a} a_{12}}{1-\mu_{a} a_{12}}}:
$$


and

$$
r_{a}^{*}=\frac{1}{a_{11}}-\mu_{a}=\begin{gathered}
\text { "net" capital productivity in the } \\
\text { capital good sector. }
\end{gathered}
$$

Consider now an alternative technique $b$, again producing two goods, the same consumption good as before, but a different capital good with depreciation rate $\mu_{b}$. Technique $b$ is assumed to use only the second type capital good (but the same primary factor labor). Denoting the fixed coefficients for this technique by $b_{i j}$, we can write analogous expressions leading to the equation for the FPC for this case (see $F P C_{b}$ in Figure I):

$$
w=\frac{1-\left(\mu_{b}+r\right) b_{11}}{b_{02}+\left(\mu_{b}+r\right) G_{b}}
$$

Suppose our hypothetical economy must select one of two alternative techniques. There is one switching point $S$ corresponding to the critical rate of interest $r_{o}$. Technique $b$ is more profitable for $0 \leq r<r_{o}$, and technique $a$ is more profitable for $r_{o}<r<r_{a}^{*}$; thus the factor price frontier is the broken curve $w_{b}^{*} S r_{a}^{*}$. The techniques are ordered by the rate of interest because as $r$ falls below $r_{o}$ and the economy switches from technique $a$ to technique $b$, technique $a$ will never recur if $r$ falls further. But is this feature inherent in the model? The answer turns out to be negative even in this highly simplified model. Figure II illustrates a case where the

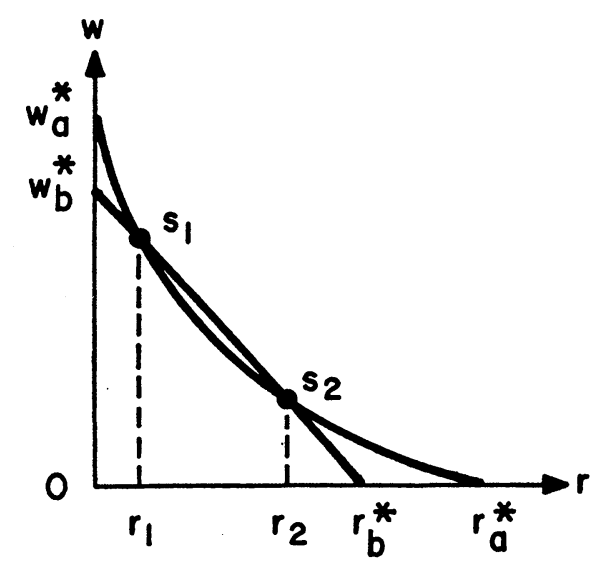

FIGURE II

\footnotetext{
(When $\left.\mu_{a}=0, w_{a}^{*}=\frac{1}{a_{02}}\right) . w_{a}^{*}$ will also correspond to the total consumption per capita in a stationary state, a fact that we shall use in subsequent discussion.
} 
two curves cross twice. When $r$ becomes less than $r_{1}$, the economy switches back to technique $a$. Technique $a$ is profitable for two disjoint intervals of $r, 0<r<r_{1}$ and $r_{2}<r<r_{a}^{*}$, and the two techniques cannot be ordered. That either one of the cases is in general possible can readily be seen by equating $w$ in equations (III.3) and (III.4) and solving for $r$. We obtain a quadratic equation for $r$ which, in principle, can easily have two roots in the positive quadrant. A condition for that occurrence can be formulated in terms of the coefficients $a_{i j}$ and $b_{i j}$. Similarly we can use some known method, such as Descartes' rule of signs, to determine a sufficiency condition which prevents that occurrence. In the next section we shall take the latter approach in the discussion of the general $n$ sector case. Here a more straightforward and economically meaningful condition can be formulated. (The latter does not, unfortunately, hold in the $n$-sector case.)

Since we know that there are at most two switching points in this simplified model, we can state the following obvious sufficiency condition for unique switching:

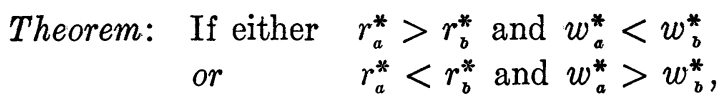

then there exists only one switching point in the positive quadrant.

In other words, whenever the technique which has a higher capital/output ratio in the capital good industry is also more laborproductive in the consumption good industry, then these techniques can be ordered in an unambiguous manner. (See, e.g., Figure I.)

A number of remarks are now in order:

1. The class of cases which are excluded by this sufficiency condition includes:

(a) Cases in which no switching point exists (i.e., where one technique completely dominates the other).

(b) Cases with multiple roots or cases in which the curves cross only at end points (i.e., $w_{a}^{*}=w_{b}^{*}$ or $r_{a}^{*}=r_{b}^{*}$ ). These again are cases in which one technique can be ignored since it is dominated.

Both (a) and (b) can be classified as irrelevant since the FPF (envelope) is unchanged by their exclusion.

(c) Cases which have two separate crossings inside the positive quadrant, as illustrated in Figure II..$^{5}$

5. After we had completed our analysis, we became aware of the recent book by Professor John R. Hicks, Capital and Growth (Oxford: Oxford University Press, 1965); there he has discussed this two-sector model and the reswitching phenomenon. The sufficiency condition which Hicks derives for 
2. This sufficiency condition applies to an economy with any number of alternative two-sector techniques all using different capital goods and having the same properties as $a$ and $b$, if the condition of the theorem holds for any pair of techniques $a$ and $b$. It is sufficient for no reswitching that the ordering of techniques by $r^{*}$ is exactly the reverse of the ordering by $w^{*}$.

3. This sufficiency condition is not a necessary condition for nonswitching.

Figure III illustrates an example with three techniques (1, 2 and 3) where each pair satisfies the sufficiency condition; yet technique 2 is irrelevant since it is dominated by the combination of 1

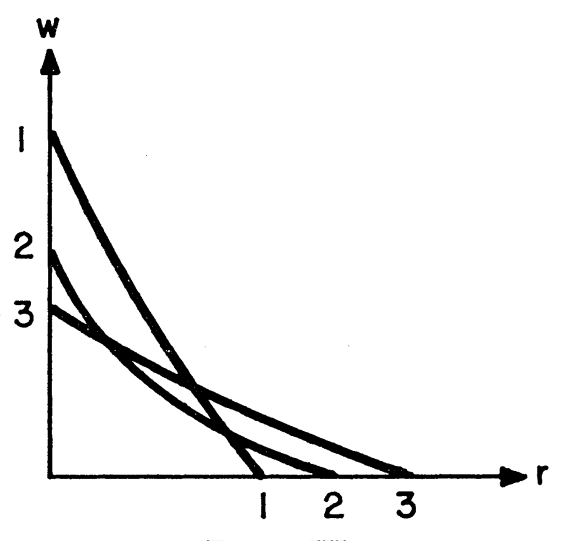

Fraure III

and 3. Figure VI in Section V illustrates a case where the sufficiency condition is not satisfied, but nevertheless each technique appears only once on the FPF envelope. This case is particularly interesting because we can use it to show that behavior which is "perverse" from the point of view of classical capital theory can occur even when there is no reswitching. We shall return to this point later. ${ }^{6}$

4. We have thus far assumed that $G \neq 0$ for all techniques. Obviously reswitching can also occur when $G=0$ for some but not all techniques. When $G=0$ for all techniques, factor proportions in the two industries are equal. Then the $F P C$ 's are straight lines and the system degenerates to Samuelson's simple surrogate capital model ${ }^{7}$ in which reswitching obviously cannot occur.

nonswitching is that the ratio of factor intensities must be the same for all techniques (see p. 154). However, this condition is overly strong since it also includes cases of complete dominance which are irrelevant.

6. See Sec. V below and Pasinetti, op. cit., fn. 14 .

7. Samuelson, op. cit. 
5. It should be stressed that the model discussed above is extremely simplified and that the sufficiency condition given does not lend itself to easy generalization if activities use more than one capital good. The latter fact can be seen by considering a case with one consumption good and two capital goods where prices are clearly equations of the third degree. Thus in general there may be three switching points and we can no longer formulate any simple sufficiency condition for nonswitching. We shall return to this problem in the next section.

An interesting question arises at this point. How crucial is the assumption that the two (or more) capital goods in our simplified model are different, i.e., nontransferable between activities? Can we get reswitching if all activities use the same capital good? The answer to this question turns out to be negative, and we have the following theorem:

Theorem: In a two-sector economy with many alternative independent techniques for producing the two goods, if there is only one capital good in the system, reswitching cannot occur.

Proof: Since there is only one capital good (let it be of type a), there is only one $P_{K}$ in the system. From equation (III.1) we can see that for any given $r$, there will be only one most efficient activity for producing the one capital good and it cannot recur. Looking at the factor-price frontier in the $\frac{w}{P_{K}}-r$ plane, we find an envelope of straight line segments. We now need only show that within the range of $r$ for any one such segment, an activity for the production of the consumption good cannot recur. This problem, however, is equivalent to that of finding switching points in the previous model for the case in which there is only one way of producing the capital good. From previous considerations we can immediately deduce that for any two FPC's in the $w-r$ plane, there can be at most one switching point in the range $0<r<r_{a}^{*}$ (because there is already one common intersection at $r_{a}^{*}=\frac{1}{a_{11}}-\mu_{a}$ ). Thus even though a single activity for producing the consumption good may recur, in this case we cannot have the simultaneous recurrence of two activities (for the two goods). Thus reswitching in the sense of Levhari cannot occur. Q.E.D.

The above theorem also holds for a multisector economy pro- 
ducing many intermediate (or consumption) goods as long as there is only one capital good (i.e., only one interest earning asset) ${ }^{8}$

All the above examples fail to meet Levhari's indecomposability assumption and thus may be deemed irrelevant for his nonswitching theorem. (Note that our examples are decomposable because the consumption good uses capital goods in its production but is not itself used in the production of capital goods.) ${ }^{9}$

For the sake of completeness, we end this section with a numerical example as a definite proof that reswitching can occur despite indecomposability. Although we do not think that indecomposability has any relevance to the switching problem, it is pedagogically preferable and logically crucial to choose an example which satisfies every assumption of the theorem to be disproved. ${ }^{1}$ The following is a two-sector indecomposable example with circulating capital (i.e., $\mu=1$ ) in full conformity with Levhari's model:

The economy produces two goods labeled 1 and 2 . Good 1 has two alternative activities while good 2 can be produced by only one available activity:

\begin{tabular}{lccc}
\hline & \multicolumn{2}{c}{ Sector 1} & Sector 2 \\
\cline { 2 - 3 } & Activity 1 & Activity 2 & \\
\hline Labor & 0.66 & 0.01 & 1.0 \\
Good 1 & 0.3 & 0 & 0.1 \\
Good 2 & 0.02 & 0.71 & 0 \\
\hline \hline
\end{tabular}

Thus there are two possible techniques of production:

and

$$
\text { technique } a=\left[\begin{array}{l}
a_{o} \\
a
\end{array}\right]=\left[\begin{array}{ll}
0.66 & 1.0 \\
\hline 0.3 & 0.1 \\
0.02 & 0
\end{array}\right]
$$

$$
\text { technique } b=\left[\begin{array}{l}
\frac{b_{o}}{b}
\end{array}\right]=\left[\begin{array}{ll}
0.01 & 0.1 \\
\hline 0 & 0.1 \\
0.71 & 0
\end{array}\right] \text {. }
$$

Notice that both techniques are indecomposable.

Some values of $\frac{P_{1}}{w}$ and $\frac{P_{2}}{w}$ for both techniques are computed in Table I.

8. This is the obvious case in which the concept of "capital-intensity" can be defined unambiguously.

9. Nothing, however, is changed in our analysis if the consumption good is also used as an intermediate good in the Leontief sense; then the technique matrix may appear indecomposable. But this is obviously not the kind of indecomposability that Levhari had in mind.

1. Pasinetti's numerical example is thus, strictly speaking, not a valid counterexample to Levhari's theorem. 
TABLE I

\begin{tabular}{|c|c|c|c|c|c|}
\hline \multirow{2}{*}{$\lambda=\frac{1}{1+r}$} & \multirow[b]{2}{*}{$r$} & \multicolumn{2}{|c|}{ Technique $a$} & \multicolumn{2}{|c|}{ Technique $b$} \\
\hline & & $\frac{P_{1}}{w}$ & $\frac{P_{2}}{w}$ & $\frac{P_{1}}{w}$ & $\frac{P_{\mathbf{2}}}{w}$ \\
\hline 1. & 0. & 0.9742 & 1.0974 & 0.7750 & 1.0775 \\
\hline 0.95 & 0.053 & 1.0512 & 1.1633 & 0.8653 & 1.1437 \\
\hline 0.90 & 0.111 & 1.1413 & 1.2379 & 0.9729 & 1.2192 \\
\hline 0.85 & 0.176 & 1.2481 & 1.3233 & 1.1028 & 1.3062 \\
\hline 0.80 & 0.250 & 1.3769 & 1.4221 & 1.2619 & 1.4077 \\
\hline 0.75 & 0.333 & 1.5350 & 1.5380 & 1.4598 & 1.5279 \\
\hline 0.70 & 0.429 & 1.7338 & 1.6763 & 1.7112 & 1.6730 \\
\hline 0.65 & 0.538 & 1.9911 & 1.8448 & 2.0384 & 1.8521 \\
\hline 0.60 & 0.667 & 2.3371 & 2.0562 & 2.4775 & 2.0796 \\
\hline 0.55 & 0.818 & 2.8266 & 2.3321 & 3.0907 & 2.3801 \\
\hline 0.50 & 1.000 & 3.5714 & 2.7143 & 3.9944 & 2.7989 \\
\hline 0.45 & 1.222 & 4.8397 & 3.2977 & 5.4335 & 3.4296 \\
\hline 0.40 & 1.500 & 7.4737 & 4.3684 & 8.0225 & 4.5056 \\
\hline 0.35 & 1.857 & 16.1936 & 7.4839 & 13.8544 & 6.8155 \\
\hline
\end{tabular}

It is seen that technique $b$ is selected for very low and for very high rates of interest, while for interest rates in the interval (approximately) $r=0.45$ to $r=1.79$, technique $a$ is optimal.

\section{Reswitching in a General Capital Model}

We now focus our attention on a general model with $n$ capital goods and examine the reswitching phenomenon for this case. Obviously, if reswitching can occur in special two-sector technologies, it will be the rule rather than the exception with any larger number of sectors. We have investigated reswitching where there are a number of alternative activities to produce each good. Subsequently we discuss a general sufficiency condition for nonswitching which, as expected, is very restrictive and is most probably not a realistic assumption in any practical situation.

Consider, as in Levhari's analysis, a general model of an economy using one primary good, labor, and producing $n$ (capital) goods, each one of which can be produced by $k_{i}$ alternative activities $(i=1,2, \ldots, \mathrm{n})$. We thus have II $_{i=1}^{n} k_{i}$ alternative technique matrixes $\left[\begin{array}{c}a_{o} \\ \dot{a} \\ \dot{a}\end{array}\right],\left[\begin{array}{c}b_{o} \\ \dot{b}\end{array}\right], \ldots . .$, which constitute the economy's "book of blue prints" or its technology. To make our discussion sufficiently general, suppose these $n$ goods can be fixed- 
capital goods. Then, for example, prices using technique $a$ are given by the vector equation

$$
\frac{P}{w}=a_{o}[\mathrm{I}-(\mu+r) a]^{-1}=p_{a}(r) \text {, where }(\mu+r) \text { is a }
$$

diagonal matrix with the element in the $i$-th row and $i$-th column equal to $\left(\mu_{i}+r\right) .^{2}$ From the subsequent analysis it will be clear that everything discussed for this general model is a fortiori applicable to the Levhari-Sraffa model with circulating capital. ${ }^{3}$

We will find it convenient to express the price vector in the following form:

$$
p_{a}(r)=\frac{T_{a}(r)}{Q_{a}(r)}
$$

where $T_{a}(r)$ is a vector of polynomials, each of degree at most $(n-1)$, and $Q_{a}(r)>0$ is a polynomial of degree $n .^{4}$

Suppose that technique $a$ is preferred to any other technique $b$ for $r_{1}<r<r_{o}$, and suppose that $r=r_{1}$ is a genuine switching point (i.e., ties are excluded). For the open interval $r_{1}<r<r_{o}$, we must have

$$
p_{a}(r) \leq p_{b}(r),
$$

from which it follows that

Now define

$$
p_{a}(r)(\mu+r)[a-b]+\left(a_{o}-b_{o}\right) \leq 0.5
$$

Note that $G(r)$ is a vector of polynomials whose elements denoted by $G_{i}(r)$ are linear combinations of the polynomials $T_{a}(r)$ and $Q_{a}(r)$; thus $G_{i}(r)$ is in general a polynomial of degree $n$.

We next observe that the $i$-th column of $[a-b]$ will consist of either zeros or a mixture of both positive and negative (and possibly zero) elements. The same applies to the augmented matrix when labor inputs are included. ${ }^{6}$ It follows that for all $r$ such that

2. We note that if, as in the two-sector model, there is also an $(n+1)$ th good which uses all other inputs but is not used by any other activity, its price equation will be $\frac{P_{n+1}}{w}=a_{o},{ }_{n+1}+(\mu+r) p_{a}(r) a_{n+1}$.

3. Observe that $\mu_{i}=1, i=1,2, \ldots, n$, is simply a special case.

4. (IV.2) may be easily derived from (IV.1) by using the familiar adjoint method to calculate the inverse of $[I-(\mu+r) a] . Q_{a}(r)$ is equal to det $[I-(\mu+r) a]$. Since $[I-(\mu+r) a]$ has rank $n$, it is a polynomial of degree $n$. Every $(n-1) \times(n-1)$ minor of $[I-(\mu+r) a]$ is a polynomial of degree at most $(n-1)$, and every component of the vector $T_{a}(r)$ is a linear combination of the latter polynomials and hence also of degree at most $(n-1) . Q_{a}(r)$ $>0$ by the Hawkins-Simon condition.

5 . If there were only circulating capital, $(\mu+r)$ would be replaced by $(1+r)$ in (IV.3), an inequality obtained by Levhari, op. cit., p. 104.

6. The case of an all (semi) positive or all (semi) negative column can be excluded since it would indicate that one of the matrixes has been chosen inefficiently, i.e., it is dominated by some other matrix for all $r$. 
$r_{1}<r<r_{o}$ and for each $i$, we must have either $G_{i}(r) \equiv 0$ or $G_{i}(r)$ $<0$. An intermediate case in which $G_{i}(r) \leqslant 0$ (e.g., a multiple root inside the interval) can be ruled out by making the interval $r_{1}<r$ $<r_{o}$ small enough. A candidate for switching must obviously come from an activity (or activities) for which $G_{i}(r)<0$.

Since $r_{1}$ is defined as a switching point, there exists at least one index, say $i=q$, and a vector $\left[\begin{array}{c}b_{o} \\ \dot{b}\end{array} \cdot\right]_{q}$ for which

$$
G_{q}\left(r_{1}\right)=0 \text { and } G_{q}(r)>0
$$

for some interval $r_{2}<r<r_{1}$. (The latter follows from the polynomial property of $G$.) ${ }^{7}$

If $q$ is the only index for which (IV.5) holds, we can readily see that our choice of the new technique $b$ (at least for the interval $\left.r_{2}<r<r_{1}\right)$ will be a change of $q$-th activity from $\left[\begin{array}{c}a_{o} \\ \cdot \dot{a}\end{array}\right]_{q}$ to $\left[\begin{array}{l}b_{o} \\ \dot{b}\end{array}\right]_{q}$, while all other activities remain the same as those in $\left[\begin{array}{c}a_{o} \\ \dot{a}\end{array}\right]$. Without loss of generality we can assume $q=1$ and write

$$
\begin{aligned}
& {\left[\begin{array}{c}
a_{o} \\
\cdot a
\end{array}\right]-\left[\begin{array}{l}
b_{o} \\
\cdot b \\
b
\end{array}\right]=\left[\begin{array}{l}
d_{o} \\
\dot{d}
\end{array}\right]}
\end{aligned}
$$

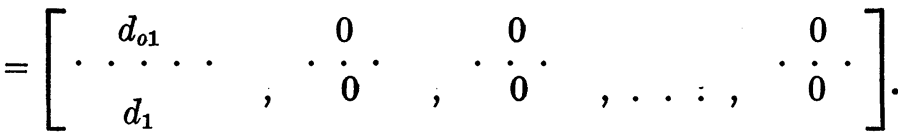

Thus for all $r$ such that $r_{2}<r<r_{1}$ we have

$$
G_{1}(r)>0 \text { and } G_{i}(r) \equiv 0 \text { for all } i>1 \text {. }
$$

Consider the new price vector $p_{b}(r)$ given by

$$
\begin{aligned}
p_{b}(r) & =b_{o}[I-(\mu+r) b]^{-1} \\
& =\left(a_{1}-d_{o}\right)[I-(\mu+r)(a-d)]^{-1} \\
& =\left(a_{o}-d_{o}\right) R_{a}\left[I+(\mu+r) d R_{a}\right]^{-1}
\end{aligned}
$$

where $R_{a} \equiv[I-(\mu+r) a]^{-1}$.

Expanding in a power series we get:

$$
\begin{aligned}
p_{b}(r) & =\left[p_{a}(r)-d_{o} R_{a}\right]\left\{I-(\mu+r) d R_{a}\right. \\
& \left.+\left[(\mu+r) d R_{a}\right]^{2}-\ldots\right\} .
\end{aligned}
$$

7. $G_{q}(r)$, being an $n$-th degree polynomial, will have up to $n$ roots and thus in principle there will be up to $n$ switching points. By assumption $r_{1}$ is the root closest to $r_{0}$. It may be noted that here is a case where one recurring activity is synonymous with the recurrence of the entire technique matrix (suppose these are the only two matrixes available). Thus the existence of a "Ruth Cohen Curiosum" for one activity also implies the reswitching phenomenon. 
Ignoring second order terms we obtain

(IV.8)

$$
\begin{aligned}
p_{b}(r) & =p_{a}(r)-\left[p_{a}(r)(\mu+r) d+d_{o}\right] R_{a} \\
& =p_{a}(r)-\frac{G(r)}{Q a} R_{a} .
\end{aligned}
$$

Since (1) $\dot{G}_{1}(r)>0$, (2) $G_{i}(r)=0$ for $i>1$, and (3) $R_{a}>0$, we have

$$
p_{b}(r)<p_{a}(r) \text { for } r_{2}<r<r_{1}
$$

as we would expect. At the same time $\left(r_{1}-r_{2}\right)$ can be made sufficiently small so that the constructed matrix $b$ is preferable to any other matrix in that interval.

Clearly if $G_{i}(r)=0$ for more than one index $i$, say for $i=1$, . . . , $m$, then we switch $m$ activities and keep the other $(n-m)$ fixed; i.e., we take

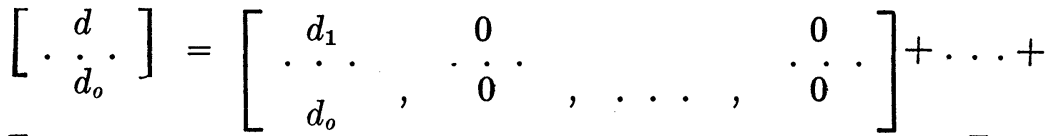

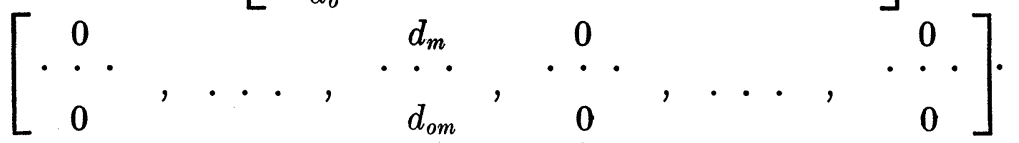

From previous considerations we find that minimizing $p_{b}(r)$ in the same interval $r_{2}<r<r_{1}$ would necessitate bringing all $m$ new activities into the matrix.

$$
\text { Since }\left[p_{a}(r)(\mu+r) d+d_{o} R_{a}\right]>0 \text { for } d_{1}, d_{2}, \ldots, d_{m},
$$

$p_{b}(r)$ in (IV.8) will be smallest if all $m$ activities are introduced simultaneously.

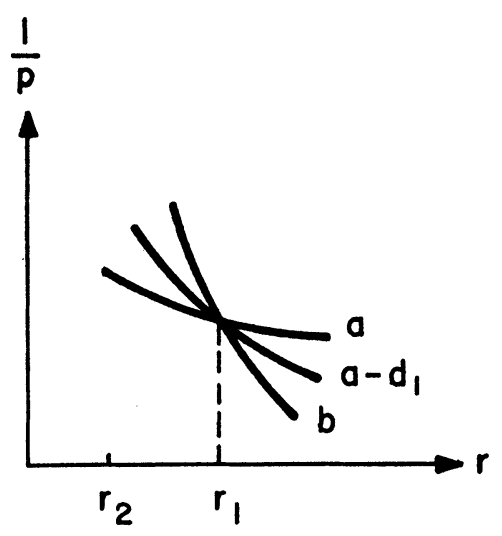

Figure IV

$r_{1}$ is a common switching point for $m$ activities when the $m$ poly- 
nomials $G_{i}(r), i=1,2, \ldots, m$, all happen to have a root at $r=r_{1}$. If by coincidence all $n$ polynomials $G_{i}(r), i=1, \ldots, n$, vanish at $r_{1}$, then and only then can we say that matrixes $a$ and $b$ which are "adjacent" on the factor-price frontier have no common column, i.e., $(a-b)$ will not contain a zero column vector. For completeness we consider the special case where $G_{s}(r) \equiv 0$ and

$$
\left[\begin{array}{c}
a_{o} \\
\dot{a}
\end{array}\right]_{s}-\left[\begin{array}{c}
b_{o} \\
\dot{b} \cdot
\end{array}\right]_{s} \neq 0
$$

for some index $i=s$. In this case prices are left unaltered by keeping $\left[\begin{array}{c}a_{o} \\ a\end{array}\right]_{s}$ in the matrix or by switching to $\left[\begin{array}{c}b_{o} \\ \cdot b \\ b\end{array}\right]_{s}$ i.e., $p_{a}(r)(\mu+r) d+d_{o} \equiv 0$ and $p_{b}(r)=p_{a}(r)$. Clearly, the above cannot hold for all $s=1,2, \ldots, n$.

Our results may be summarized by the following theorem:

Theorem: (1) In the general $n$-sector capital model there may be up to $n$ switching points between any two techniques, and thus a technique may recur up to $(n-1)$ times. (2) "Adjacent" techniques on two sides of a switching point will usually differ from each other only with respect to one activity. ${ }^{8}$ Techniques in general may differ with respect to $m$ activities $(n \geq m>1)$ only if certain $m$ independent $n$-th degree polynomials happen to have a common root at that switching point.

It should be noted that we have confined our discussion to reswitching between pairs of techniques, although the problem of reswitching as originally stated involved only intersection points which lie on the FPF envelope. It is clear that there may be no reswitching even if two or more curves intersect several times below the envelope. ${ }^{9}$ It is important to keep this distinction in mind when discussing sufficiency conditions for nonswitching; assuming that any two FPC's can intersect only once is almost certainly an overly strong sufficient condition for nonswitching. In the next section, however, it will become clear why only such strong restrictions (rather than merely nonswitching on the envelope) might ensure "classical behavior" of consumption and capital across steady states with different interest rates.

The second part of the above theorem helps to clarify discussions about the wrong step in Levhari's proof, namely his funda-

8. This particular result is also stated without proof by Pasinetti, op. cit. 9. See Figure VI in Section V below. 
mental lemma. ${ }^{1}$ His lemma is wrong, but some of the criticism about it is also wrong.

Solow has rightly pointed out that one cannot in general find a semipositive vector $x$ such that either $[a-b] x \geqslant 0,[a-b] x \leqslant 0$, or $[a-b] x=0 .^{2}$ The second part of our above theorem shows that the lemma will "almost" always work, but only trivially. In other words, we can "almost" always find $x \geqslant 0$ such that $[a-b] x$ $=0^{3}$ We agree with Pasinetti ${ }^{4}$ that despite this fact, Levhari's proof is incorrect because we could always select a vector $x$ with zeros that correspond precisely to those activities in which switching had taken place. Suppose, however, that we were to assume that there exists a vector $x$ (it can be any vector!) such that either $[a-b] x \geq 0$ or $[a-b] x \leq 0$ for any pair of matrices $a$ and $b$ with associated positive price vectors. Strangely enough, Levhari's nonswitching theorem would then be correct!

Proof: Suppose there exist two distinct switch points $r_{1}$ and $r_{2}$. Define the vector function $f(r) \equiv p_{a}(r)(\mu+r)[a-b]+\left(a_{o}-b_{o}\right)$. By assumption, $f\left(r_{1}\right)=f\left(r_{2}\right)=0$ where $r_{1} \neq r_{2}$. Also $p_{a}{ }^{\prime}(r)>0$. Now define the scalar function

$$
\phi(r) \equiv f(r) x
$$

where $\phi\left(r_{1}\right)=\phi\left(r_{2}\right)=0$. Differentiating $\phi(r)$ we obtain

$$
\phi^{\prime}(r)=\left[p_{a}(r)+p_{a}{ }^{\prime}(r)(\mu+r)\right][a-b] x .
$$

Thus for all $r \phi^{\prime}(r)>0$ if $[a-b] x \equiv 0$ and $\phi^{\prime}(r)<0$ if $[a-b] x$ $\leq 0$. Thus we cannot have $\phi\left(r_{1}\right)=\phi\left(r_{2}\right)=0$ unless $r_{1}=r_{2}$, which provides a contradiction, and hence two (or more) distinct switch points cannot exist. Q.E.D.

At first glance one might mistakenly conclude that the assumption (that there exists a vector $x$ such that $[a-b] x$ semipositive or seminegative) is only a minor restriction. However, it is a very restrictive assumption: it rules out (at least) all switches which involve only one activity and which we considered the "normal" case. $^{5}$ Economically the sufficiency condition implies (after some

1. Levhari, op. cit., pp. 104-5.

2. Any $(2 \times 2)$ numerical example in which one row of $[a-b]$ is positive and the other is negative will suffice.

3 . If $m<n$, we can always choose an $x$ with zeros in the first $m$ columns and positive elements in the remaining $n-m$ columns; thus $[a-b] x=0$. Even if $m=n$, there may be cases where the lemma is valid and thus we could claim that it is indeed valid except for extreme coincidences.

4. Pasinetti, op. cit.

5. If $[a-b]$ has one column of mixed positive and negative elements and $(n-1)$ zero columns, then there does not exist any vector $x$ such that either $[a-b] x \geqslant 0$ or $[a-b] x \leqslant 0$. This sufficiency condition can apply 
manipulation) that there exists one level of operation for which one technique uses at least as much of all inputs as the other technique and definitely more of at least one input; moreover, the latter must hold for any pair of techniques that appear on the factor-price frontier.

We end this section by presenting an alternative sufficiency condition which is again highly restrictive. The price vector for technique $a$ is given by (IV.1). For simplicity we assume that all goods depreciate at the same rate and define the scalar $\rho \equiv \mu+r$. Then

Likewise

$$
p_{a}(\rho)=a_{o}[I-\rho a]^{-1}
$$

gives the price vector when technique $b$ is used. If $\rho=\rho_{1}$ is a switching point, it follows that

(IV.11) $\quad p_{a}\left(\rho_{1}\right)-p_{b}\left(\rho_{1}\right)=a_{o}\left[I-\rho_{1} a\right]^{-1}-b_{o}\left[I-\rho_{1} b\right]^{-1}=0$.

(IV.11) is a polynomial of degree at most $n$, and a simple condition for the existence of at most one positive root can be provided by Descartes' rule of signs. The latter states that the number of positive real roots is equal to the number of variations in sign of the coefficients of the polynomial or is less than this number by a positive integer. ${ }^{6}$

We can obtain the economic interpretation of an analogous sufficiency condition by expanding (IV.9) and (IV.10) in a (convergent) vector power series:

$$
p_{a}(\rho)=a_{o}+a_{1} \rho+a_{2} \rho^{2}+\ldots
$$

where $a_{o}=a_{o}, a_{1}=a_{o} a, a_{2}=a_{o} a^{2}, \ldots$; likewise

(IV.13) $\quad p_{b}(\rho)=\beta_{o}+\beta_{1} \rho+\beta_{2} \rho^{2}+\ldots$

where $\beta_{o}=b_{o}, \beta_{1}=b_{o} b, \beta_{2}=b_{o} b^{2}, \ldots$ Thus (IV.11) becomes

(IV.14) $\quad p_{a}\left(\rho_{1}\right)-p_{b}\left(\rho_{1}\right)=\left(a_{o}-\beta_{o}\right)$

$$
+\left(a_{1}-\beta_{1}\right) \rho_{1}+\left(a_{2}-\beta_{2}\right) \rho_{1}^{2}+\ldots=0 .
$$

Note that $a_{o}$ is the vector of direct labor inputs; $a_{1}$ is the first round of indirect labor; and $a_{2}$ is the second round of indirect labor, etc. The $\beta$ 's have the same interpretation in terms of technique $b$.

Assume that there is only one sign variation in (IV.14). Assume, for example that the first term in (IV.14) is negative for the $i$-th good $\left(a_{o i} \leq \beta_{o i}\right)$, while all the other coefficients are positive

only when there are at least two nonzero columns in $[a-b]$, which in the present model, as we have seen, is not a general case. This might, however, be relevant in an 'Austrian'-type capital model, in which activities are not chosen independently.

6. See, for example, L.E. Dickson, New First Course in the Theory of Equations (New York: Wiley, 1939), p. 76. 
$\left(a_{j i} \geqslant \beta_{j i}\right.$ for $\left.j=1,2, \ldots, n\right)$. The first derivative of $p_{a}(\rho)-$ $p_{b}(\rho)$ will then be positive for $\rho>0$, and therefore (IV.14) can have at most one root. This condition on the coefficients has a simple economic interpretation. Recall that $a_{o i}$ and $\beta_{o i}$ are the direct labor inputs per unit of the $i$-th good, that $\alpha_{1 i}$ and $\beta_{1 i}$ are the first round of indirect labor needed to produce a unit of the $i$-th good, and that $a_{2 i}$ and $\beta_{2 i}$ are the second round of indirect labor needed to produce the $i$-th good, etc. ${ }^{7}$ Hence, while activity $a_{i}$ uses less direct labor than activity $b_{i}$, it embodies more indirect labor than activity $b_{i}$. If it is desired, one can substitute the word "capital" for "indirect labor," and conclude that activity $a_{i}$ is more "capital-intensive" than activity $b_{i}{ }^{8}$

We conclude that if for any pair of relevant techniques $a$ and $b$, all pairs of corresponding activities $a_{i}$ and $b_{i}$ can be ranked in terms of "capital intensity" (in the above sense, which is independent of the rate of interest), then reswitching cannot occur. ${ }^{9}$

Although the latter sufficiency condition is again highly restrictive, it may be somewhat less restrictive than the former one: note the latter allows changes of single activities while the former does not. We might also observe that the latter condition seems to be the most natural extension of our previous two-sector nonswitching theorem (see Section III). Let us again stress that, except for highly exceptional circumstances, techniques cannot be ranked in order of capital intensity. ${ }^{1}$ We thus conclude that reswitching is, at least theoretically, a perfectly acceptable case in the discrete capital model. ${ }^{2}$

Finally, let us note the crucial role of discreteness of activities

7. In the general case where the $\mu_{t}$ are unequal, the price vectors $p_{a}$ is a polynomial in $r$. One can show that the first coefficient in that polynomial will be $a_{o}^{\prime}=a_{o}\left[I-\mu_{a}\right]^{-1}=$ direct "embodied" labor input. The next terms have somewhat more complicated coefficients but have an analogous interpretation in terms of direct and indirect labor costs.

8. One can show that if we measure aggregate capital in each activity in value (or labor unit) terms, then this interpretation is precise.

The fact that the variations in signs of the input streams are related to the possibility of reswitchings (multiple roots) suggests that reswitching is similar to the problem of an investment option having more than one internal rate of return. (See also Section $V$ below.) That any number of zeros can occur in the present value function was pointed out by Samuelson, "Some Aspects of the Pure Theory of Capital," this Journal, LI (May 1937), $469-96$.

9. Note that we do not require pairs of activities to be ordered in the same way across the two matrixes, i.e., we need not have $a_{o i} \supseteqq b_{o i}$ or $a_{o i} \leqslant b_{o i}$ for all $i$.

1. We are thus in agreement with Morishima, Equilibrium, Stability, and Growth, op. cit., and Pasinetti, op. cit.

2. There is an open empirical question as to whether or not reswitching is likely to be observed in an actual economy for reasonable changes in the interest rate. 
for obtaining the reswitching result. This is best exhibited by the following interesting theorem due to both M. Weitzman and Solow: In a general $n$ capital good economy, suppose there is at least one capital good that is produced by a smooth neoclassical production function. In such an economy reswitching cannot occur provided that labor and each good are inputs in one or more of the goods produced neoclasically.

Setting the various marginal productivity conditions and supposing that at two different rates of interest the same set of inputoutput coefficients holds, the proof follows by contradiction.

\section{Some Additional Implications for Economic Theory}

\section{A. Expressions for National Product}

In order to facilitate the exposition, we assume a circulatingcapital technology, although the results can be generalized to include the fixed-capital case without difficulty. Let $X=\left(X_{1}, \ldots\right.$, $X_{n}$ ) denote the output vector. The dynamics of the model are described by

$$
X(t)-C(t)=a X(t+1),
$$

a difference equation with the interpretation that the part of this period's output which is not consumed must be equal to the input requirements for next period. We also assume that the labor supply is exogenously given, does not grow, and is fully employed. ${ }^{3} \mathrm{~A}$ steady-state solution $X(t)=X(t+1)=X$ must satisfy the balance relationship

(V.2) $\quad X=a X+C$

or

(V.2a) $\quad X=[I-a]^{-1} C$

where the labor constraint is

(V.2b) $L=a_{0} X$.

The price vector now is (as in Levhari):

(V.3) $\quad P(r)=(1+r) w a_{0}[I-(1+r) a]^{-1}$.

Manipulation of (V.2a), V.2b), and (V.3) yields the following expressions for gross national product and net national product:

(V.4a) $\quad G N P \equiv P(r) X=(1+r) w L+(1+r) P(r) a X$

and

(V.4b) $\quad N N P \equiv P(r) C=w L+r[w L+P(r) a X]$.

Since labor and circulating capital are paid for at the beginning of

3. We could also generalize the results to the case where $L$ grows according to $L / L=n$, an exogenous constant. Here we take $n=0$, and consider steady states which are in fact stationary states. 
the production period, capitalists must advance an amount equal to $[w L+P(r) a X]$, on which they earn a net profit equal to $r[w L+$ $P(r) a X]$ where $r$ is the one-period rate of interest or the profit rate.

Under the simplifying assumption that all wage income is consumed, the quantity $[w L+P(r) a X]$ has a simple interpretation. Then all saving is done by capitalists who must save-invest enough to maintain the value of circulating capital required for the steady state, namely $P(r) a X$.

Let $C^{*}$ and $C^{-}$denote capitalists' and workers' consumption, respectively. Capitalists and workers are faced with their respective budget constraints: ${ }^{4}$

$$
P(r) C^{*}=r[w L+P(r) a X]
$$

and

$$
P(r) C^{-}=w L=\text { constant. }
$$

The latter is what Samuelson has called net-net national product; it is that part of net national product left over for the primary factor (labor) after the other factors have been paid. ${ }^{5}$ Note that $C \equiv C^{*}+C^{-}$.

It is easily seen from (V.2a), (V.2b), and (V.3) that there exists a technical constraint

$$
L=P(0) C
$$

where $P(0)=a_{0}[I-a]^{-1}$. Thus for a given technique $\left[\begin{array}{c}a_{0} \\ \cdot a \\ a\end{array} \cdot\right]$, an equilibrium consumption vector $C$ must satisfy (V.7), the equation of a hyperplane; such a $C$ also satisfies (V.4b), and vice versa.

$P(r) C^{-}$does not depend on tastes because $w L$ is a fixed number and workers are free to choose any point $C^{-}$which satisfies their budget constraint (V.6) ; thus (V.6) is indeed the consumption possibility schedule for workers. But $P(r) C^{*}$ does depend on tastes. Suppose, for example, that $C^{-}$is fixed and equilibrium is established with $C^{*}=\hat{C}^{*}$. At first glance, one might think that capitalists are free to move to another point $C^{*}$ (remember that $r$ is fixed) provided that

$$
P(r) \hat{C}^{*}=P(r) \widetilde{C}^{*} \text {. }
$$

4. As we will see, it is misleading to call both (V.5) and (V.6) consumption possibility schedules. Note also that the interpretation of $C^{*}$ and $C-$ must change if the saving assumption is dropped. In fact, $C^{-}$is the maximum steady-state consumption for workers, and $C^{*}$ is the minimum steady-state consumption for capitalists. It would be an easy matter to alter the discussion which follows to include any case.

5. Paul A. Samuelson, "A New Theorem on Nonsubstitution," in H. Hegeland (ed.), Money, Growth, and Methodology; and Other Essays in Economics in Honor of Johan Akerman (Lund: C. W. K. Gleerup, 1961). 
But such a movement is in general impossible because the budget equation for capitalists (V.5) depends on $X$, and $X$ depends on $C \equiv C^{*}+C^{-}$via (V.2a). Hence (V.5) must be interpreted as a virtual consumption possibility schedule. Capitalists are actually constrained to a choice of $C^{*}$ which satisfies

$$
\begin{aligned}
\{P(r) & \left.-r P(r) a[I-a]^{-1}\right\} C^{*} \\
& =r\left\{w L+P(r) a[I-a]^{-1} C^{-}\right\} .
\end{aligned}
$$

With $r$ fixed, we conclude that $P(r) C^{*}$ and thus $P(r) C$ are not invariant to a change in tastes of either workers or capitalists, even though $P(r) C^{-}=w L=$ constant is obviously invariant. ${ }^{6}$

B. Steady-state Consumption Patterns

We now wish to consider a finite set of alternative techniques $\left[\begin{array}{l}a_{0} \\ a_{a}\end{array} \cdot\right], a=a, b, c, . .$. The fact that a technique can recur now alerts us to the existence of a "perverse case" in which a low rate of interest is not always associated with high consumption. ${ }^{7}$

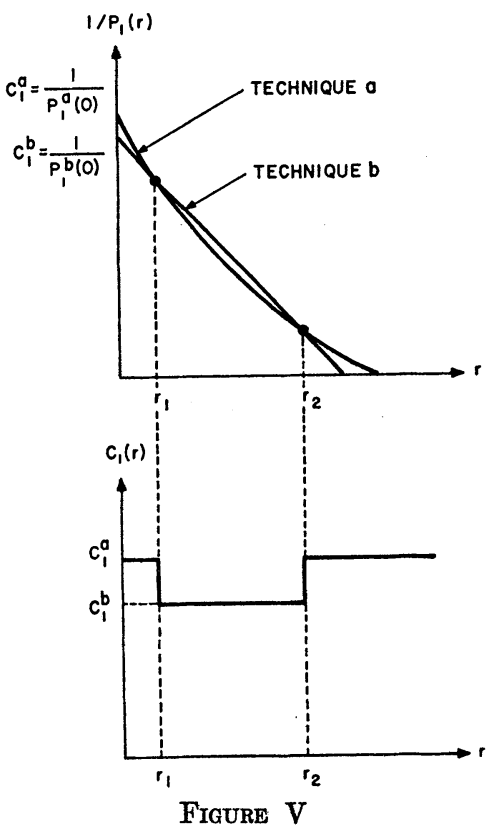

This fact may be seen most easily by setting $C_{2}=\cdots \cdot=C_{n}=0$ and observing the behavior of $C_{1}$ in different steady states. We may

6. Ibid.

7. This fact was pointed out by Morishima, Equilibrium, Stability, and Growth, op. cit., p. 126. 
solve (V.7) for

(V.9) $\quad C_{1}{ }^{a}=\frac{L}{P_{1}^{a}(0)}$

where $P_{1}{ }^{a}(0)$ is the price of good 1 when $r=0$ and technique $a$ is employed. We lose no generality by setting $w=L=1$; then the height at $r=0$ of the factor-price frontier for technique $a$ is equal to $C_{1}{ }^{a}$. A "perverse case" is illustrated in Figure V.

It is crucial to realize that while reswitching tells us that such behavior exists, the phenomenon illustrated in Figure $\mathrm{V}$ exists without reswitching, as shown in Figure VI. The cause of the "per-

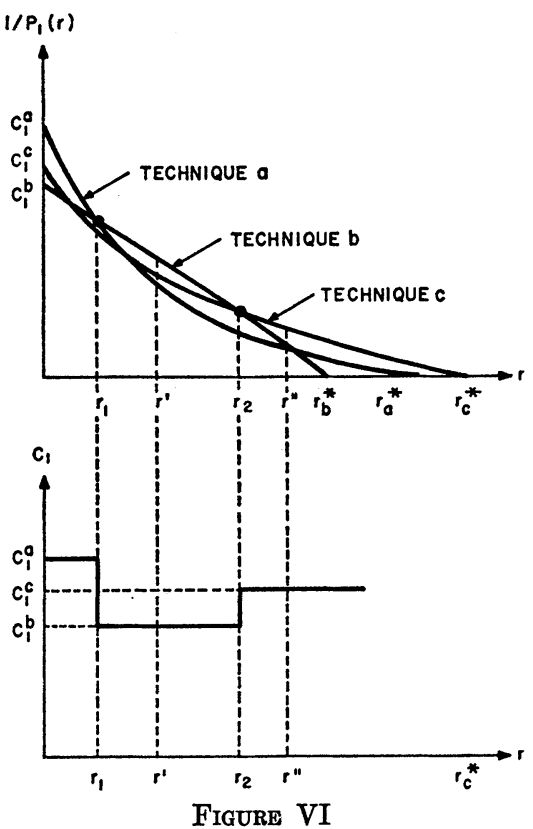

verse behavior" in Figure VI can be traced to the fact that the factor-price curves for alternative techniques cross below the outer envelope. Thus even though there are no multiple crossings on the outer envelope (the economy's factor-price frontier) and reswitching does not occur, we still find that $C_{1}$ rises when $r$ is increased from $r^{\prime}$ to $r^{\prime \prime}$ where $r_{1}<r^{\prime}<r_{2}$ and $r_{2}<r^{\prime \prime}<r_{c}^{*}$.

We note that the sufficiency conditions for nonswitching which we have stated and proved in Sections III and IV may be, in general, overly strong to preclude reswitching on the FPF envelope. However, these same conditions may be necessary if the monotonicity of $C(r)$ is to be preserved. 
The problem is a bit harder to analyze when there is positive consumption of more than one good, but the same qualitative behavior is possible. With alternative techniques the hyperplane equation (V.7) becomes a set of equations:

$$
L=P^{a}(0) c^{a}, a=a, b, c, \ldots .
$$

For a two-good economy (V.10) become the equations of straight lines in the $C_{1}-C_{2}$ plane, as illustrated in Figure VII for alter-

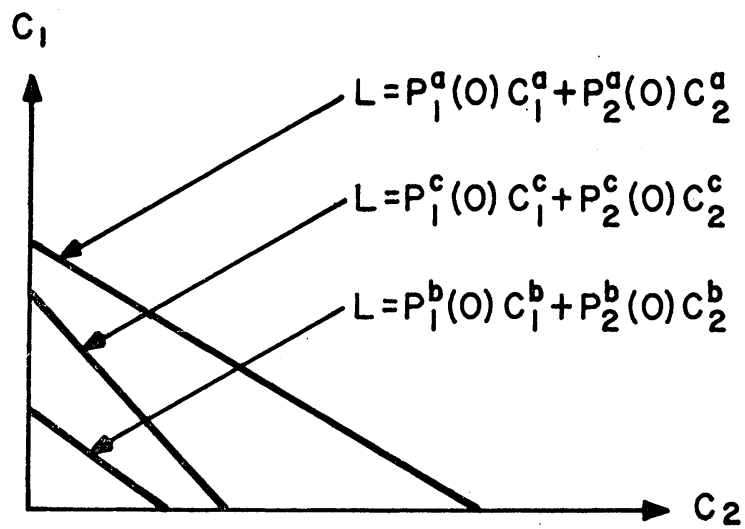

FIGURE VII

native techniques $a, b$, and $c .^{8}$ If, as in Figure VI, technique $a$ is used for $0 \leqslant r \leqslant r_{1}, b$ for $r_{1} \leq r \leq r_{2}$, and $c$ for $r_{2} \leq r \leq r_{c}^{*}$ where $r_{1}<r_{2}$, we can unambiguously say that steady-state consumption is high for low $r$, then lower for higher $r$, but higher again for still higher $r$.

Other conclusions follow from the above analysis. First, since (1) the nonsubstitution theorem tells us that the real wage in terms of every good is always maximized for a given $r$, and (2) the economy's factor-price frontier is downward sloping, it follows that consumption is maximized when $r=0$, although that maximum may not be unique. ${ }^{9}$ Second, workers' consumption is always higher for lower $r$, a conclusion which follows immediately from (V.6) and the fact that prices always increase when $r$ is increased. Finally, the steady-state value of circulating capital $P(r) a X$ is not a

8. The three lines in Figure VII are parallel if we have the Marx case of "equal organic composition of capital." The nonsubstitution theorem guarantees that the lines can never cross because all prices are simultaneously minimized.

9. If we were to assume $L / L=n>0$, per capita consumption would be maximized when the Golden Rule condition $r=n$ is satisfied. 
monotonic function of $r$, a fact also stated by Pasinetti, Morishima, and others. ${ }^{1}$

\section{Transitions between Steady States}

We have completely ignored how the economy moves from one steady state to another. It is as if there were different planets possessing the same book of blueprints (set of techniques) but which were in different steady states that correspond to different exogenously given $r$ 's. We then would observe each planet and compare their steady-state equilibriums. To discuss a movement from one steady state to another would in general require a theory of interest rate determination. Moreover, we would need to examine dynamic motions of the system and stability problems might become important.

There is, however, a special case which is illuminating and which we can easily discuss. Suppose that the exogenously given interest rate is a switch point between techniques $a$ and $b$; then both techniques are viable at the given interest rate and $P^{a}(r)=P^{b}(r)$. The economy can usually move from consumption vector $C^{a}$ to $C^{b}$, although we shall ignore the exact mechanism by which the movement is in fact accomplished. The above problem has been discussed by Solow, and he has proved that the social rate of return to saving, $\rho$, is equal to the switch-point interest rate $r$; a brief discussion of Solow's proof follows.

Suppose that the economy initially uses only technique $a$ and has a corresponding consumption vector $C^{a}$. If the economy is able to move in one period to an equilibrium where only technique $b$ is used, there must exist a consumption vector $\bar{C} \geqslant 0$ which satisfies

$$
X=\bar{C}+b Y
$$

where $b Y$ is the vector of input requirements using technique $b .^{2}$ If such a $\bar{C}$ can be found, the economy may consume $\bar{C}$ for one period and then move into steady-state equilibrium with technique $b$ and consumption $C^{b}$. Defining the social rate of return

$$
\rho \equiv \frac{P\left(C^{b}-C^{a}\right)}{P\left(C^{a}-\bar{C}\right)},
$$

Solow proved that $\rho=r .^{3}$ op cit.

1. Morishima, Equilibrium, Stability, and Growth, op. cit., and Pasinetti,

2. Solow has also considered a more complicated case in which a oneperiod transition is impossible because there does not exist a solution to $X=$ $\bar{C}+b Y$ with $\bar{C} \geqq 0$. The conclusion $\rho=r$ may remain valid even when more than one period is required to complete the transition.

3. From (V.4a) and (V.4b) we have $P C^{a}=(1+r) w L+r P a X, P C^{b}=$ 


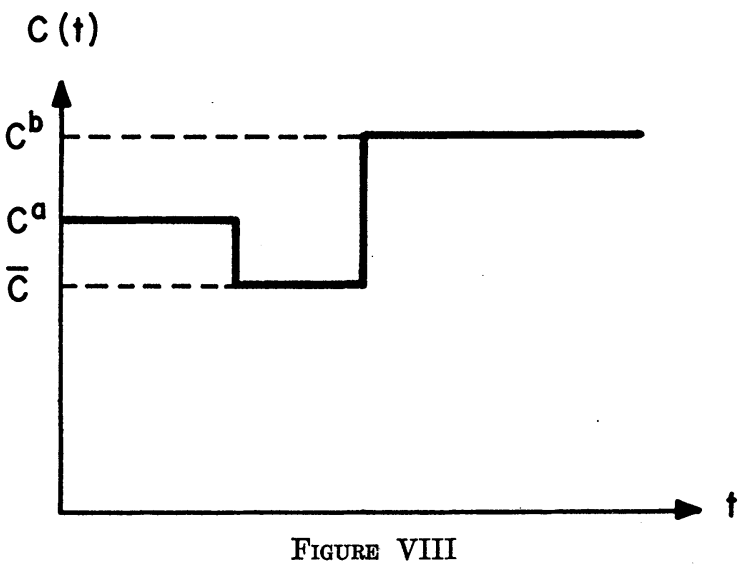

In a well-behaved case $C^{a}>\bar{C}$ and $C^{b}>C^{a}$, and the time path of consumption appears as in Figure VIII. But in a "perverse" case, $C^{a}<\bar{C}$ and $C^{b}<C^{a}$ as illustrated in Figure IX.

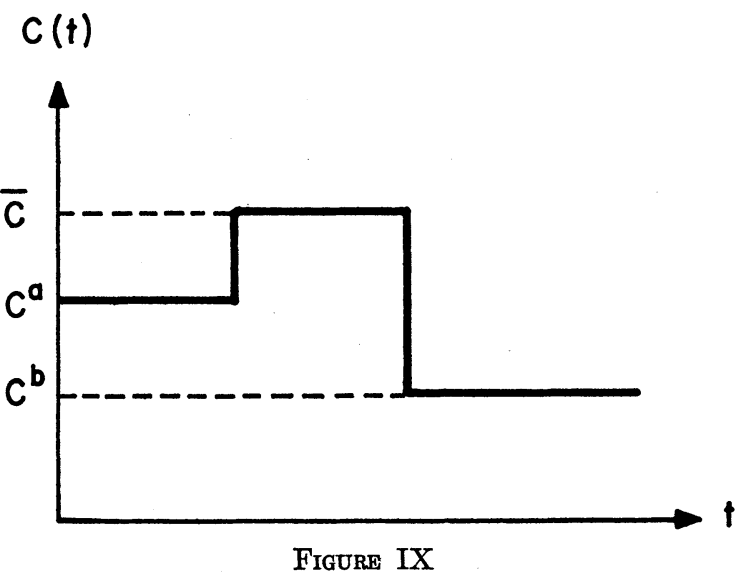

What can be concluded?

Since $P^{a}(r)=P^{b}(r)$, workers are not affected by the change: they may still consume $C^{-}$where $P C^{-}=w L$. But the story is not the same for capitalists. As $C \equiv C^{*}+C^{-}$, it is $C^{*}$ which changes when the economy moves from technique $a$ to $b$. If $C^{* b}<C^{* a}$, can we conclude that capitalists have been hurt? The answer is most

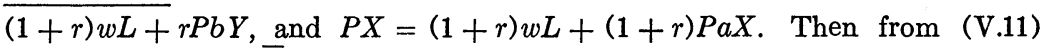
we obtain $P C^{a}-P \bar{C}=P b Y-P a X$. Likewise, $P C^{b}-P C^{a}=r(P b Y-P a X)$, and the conclusion $\rho=r$ follows immediately. 
certainly "no," for it is the capitalists who enjoyed a splash of consumption $\bar{C}^{*}>C^{* a}$, and the extra consumption in which they indulged for one period is exactly equal to the value of their foreverlower consumption stream $C^{* b}<C^{* a}$ discounted at the social rate of return. ${ }^{4}$

Finally let us point out a corollary of Solow's theorem. If there are a number of switching points, then at all of them the rate of interest (usually) equals the rate of return to saving. The physical acts of moving back and forth from one consumption "plateau" to the other as the rate of interest changes will be identical at every switch point, but the interest rate is different at each switch point. In other words, here is a clear illustration of the analogy between the reswitching problem and the existence of multiple rates of return in investment profitability calculations.

One may recall that we have been warned long ago that "there is no new thing under the sun." 5

Massachusetts Institute of Technology

and Hebrew University, Jerusalem

University of Pennsylvania

Massachusetts Institute of Technology and

HARVARD UNIVERSITY

4. The above argument depends on keeping $r$ fixed. As pointed out by Samuelson, op. cit., when $r=0$, workers get all of NNP with $P(O) C-=$ $P(O) C$ and $C^{*}=0$; and when $r=r^{*}$, the maximum rate of interest where $w=0, P(0) C^{*}=P C$ and $C^{-}=0$.

5. Bible, Ecclesiastes 1.9 (about B.C. 977). 